\title{
Contracción, hinchamiento y peso específico aparente de madera de eucalipto colorado (Eucalyptus tereticornis Smith)
}

\section{Shrinkage, swelling and apparent specific gravity of red gum (Eucalyptus tereticornis Smith)}

\author{
Cardoso, Andrea ${ }^{(1)}$, Cagno, Matías ${ }^{(1)}$, Cárdenas, Patricia ${ }^{(1)}$, Gatto, Francisco ${ }^{(1)}$ \\ (1) Departamento de Proyectos Forestales, Laboratorio Tecnológico del Uruguay, LATU, Uruguay \\ Contacto: acardoso@latu.org.uy
}

Recibido: 12/6/2013 - Aprobado: 30/10/2013

\begin{abstract}
$\underline{\text { Resumen }}$
Con el objetivo de contribuir al conocimiento del comportamiento de la madera de Eucalyptus tereticornis en Uruguay, se estudió la contracción y el hinchamiento lineal y volumétrico para diferentes condiciones de humedad relativa, a una temperatura de $25^{\circ} \mathrm{C}$. Se construyó una isoterma parcial dándole importancia a las condiciones de humedad de equilibrio en las que es posible encontrar productos realizados con esta madera en Uruguay, por ejemplo, pisos interiores o exteriores.

De cinco árboles de una cortina de 50 años ubicada en el departamento de Durazno se seleccionaron discos a dos alturas. A partir de estas se prepararon probetas para las determinaciones de contracción e hinchamiento. Se encontró que el peso específico aparente básico fue de 0,630 , al $12 \%$ de contenido de humedad fue de 0,859 y al $15 \%$ de 0,872 . La contracción radial, tangencial y volumétrica fue de $7,04 \%, 14,70 \%$ y $20,64 \%$, respectivamente, y el hinchamiento radial fue de $7,61 \%$, el tangencial de $17,61 \%$ y $26,62 \%$ el volumétrico. Sobre la isoterma se observó una diferencia esperable entre la curva de sorción y la de desorción en el rango de humedad relativa estudiado de $53 \%$ a $84 \%$.

Palabras clave: Histéresis, humedad de equilibrio, isoterma, densidad.
\end{abstract}

\begin{abstract}
With the aim of contributing to the knowledge of the behavior of Eucalyptus tereticornis wood in Uruguay, a study of linear and volumetric shrinkage and swelling at different conditions of relative humidity at a temperature of $25{ }^{\circ} \mathrm{C}$ was made. From these data, a partial isotherm was built giving importance to the equilibrium moisture conditions in which it is possible to find products with this wood, e.g. floors and decks.

Five trees from a 50 years old shelter belt located in Durazno were randomly selected. Discs were collected at two heights in each tree from which specimens were prepared for shrinkage and swelling test. It was found that the basic apparent specific gravity was $0,630,0,859$ at $12 \%$ and 0,872 at $15 \%$ of moisture content. The radial, tangential and volumetric shrinkage was $7,04 \%$, $14,7 \%$ and $20,64 \%$, respectively, and the radial, tangential and volumetric swelling was $7,61 \%, 17,61 \%$ and $26,62 \%$. It was observed an expected difference between the curve of sorption and desorption in the studied range of relative humidity from $53 \%$ to $84 \%$.
\end{abstract}

Keywords: Hysteresis, equilibrium moisture, isotherm, density.

\section{Introducción}

En Uruguay, aunque actualmente es posible encontrar madera de Eucalyptus tereticornis casi únicamente en cortinas de abrigo y sombra (MGAP-DGF, 2012), la madera de esta especie es considerada como apta para la obtención de productos de alto valor agregado, así como para la fabricación de pisos, escalones, marcos de puertas y ventanas, en construcciones pesadas y estructuras como soportes enterrados, paneles para pisos, vigas, entre otros (Bootle, 1983; Boas, 1947; FWPA, 2013).

El análisis del comportamiento de la madera de esta especie, frente a las diferentes condiciones ambientales en las que es posible encontrarla en nuestro país, es una necesidad para mejorar su uso y evitar que se produzcan deformaciones y grietas en los productos. Este trabajo es de importancia para la caracterización respecto a la estabilidad dimensional de la madera proveniente de árboles añosos, ya que es el material que principalmente se puede encontrar de $E$. tereticornis en las carpinterías y aserraderos nacionales.

En la actualidad, la madera de esta especie se utiliza en Uruguay principalmente en productos de carpintería rural, y al conocer su peso específico, contracción e hinchamiento es posible un incremento en la generación de productos de alto valor agregado.

Este estudio se plantea como objetivo la caracterización de la contracción e hinchamiento radial y tangencial de la madera de $E$. tereticornis y la elaboración de la isoterma higroscópica a $25^{\circ} \mathrm{C}$, enfocándose en las condiciones ambientales más comunes en las que es posible encontrar esta madera en Uruguay. Asimismo, este trabajo 
pretende contribuir al conocimiento de la madera de la especie en su aspecto tecnológico para mejorar su uso comercial.

Existen antecedentes de datos generados sobre esta especie en el país y en el resto del mundo, en algunas propiedades físicas como el peso específico, la contracción (Bootle, 1983; Coronel, 1994; Mantero, 1999; FWPA, 2013) y el hinchamiento (Coronel, 1994), así como algunas propiedades mecánicas de la madera (dureza, flexión estática y compresión) y durabilidad natural (Bootle, 1983; Mantero, 1999; FWPA, 2013).

Considerar la estabilidad dimensional de cada madera es fundamental para adecuar los usos de los productos maderables. Autores como Coronel (1994) realizan una clasificación de los usos posibles para las maderas según los coeficientes de contracciónhinchamiento. Etchenique et al. (1975) y Ordóñez et al. (1990) también generaron criterios para clasificar la estabilidad dimensional de la madera. Las categorías de cada una se presentan en la Tabla 1.

\begin{tabular}{|c|c|c|}
\hline Clasificación & Categoría & $\begin{array}{c}\text { Coeficiente de } \\
\text { anisotropía }\end{array}$ \\
\hline \multirow{2}{*}{$\begin{array}{c}\text { Según usos posibles } \\
\text { (Coronel, 1994) }\end{array}$} & Excelente & $1,2-1,5$ \\
\cline { 2 - 3 } & Normales & $1,6-1,9$ \\
\cline { 2 - 3 } & Con dificultades & $>2,0$ \\
\hline $\begin{array}{c}\text { Según índice de } \\
\text { distorsiones y alabeos } \\
\text { (Etchenique et al., 1975) }\end{array}$ & Baja & $1,0-1,7$ \\
\cline { 2 - 3 } & Alta & $1,7-2,3$ \\
\hline \multirow{2}{*}{$\begin{array}{c}\text { Según la estabilidad } \\
\text { dimensional } \\
\text { (Ordóñez et al., 1990) }\end{array}$} & Muy alta & $>2,3$ \\
\cline { 2 - 3 } & Estable & $<1,5$ \\
\cline { 2 - 3 } & Moderadamente estable & $2,0-2,5$ \\
\cline { 2 - 3 } & Inestable & $2,5-3,0$ \\
\cline { 2 - 3 } & Muy inestable & $>3,0$ \\
\hline
\end{tabular}

Tabla 1. Criterios de clasificación según coeficiente de anisotropía (CT/CR).

Debido a la heterogeneidad que presenta el material es necesario su análisis en el radio, es decir, de médula a corteza, y en altura. Coronel (1994) indica que los valores de contracción e hinchamiento son mayores en la medida que aumenta el peso específico aparente. Calvo (2006) obtiene resultados que condicen con esta tendencia para árboles más jóvenes (17 años) de Eucalyptus grandis con un peso específico aparente anhidro de 0,562 .

Por otro lado, en un estudio realizado a partir de árboles de Eucalyptus bosistoana de 42 años cultivados en Uruguay se observó una tendencia diferente a la indicada por los autores citados, en la cual el peso específico aparente básico crece pero la contracción radial y tangencial disminuyen hacia una posición cercana a la corteza (Cardoso y Castagna, 2012).

Kollmann y Coté (1984), remitiendo a varios autores, relacionan la variación transversal de la contracción de la madera con las alternancias de los incrementos de leño temprano-tardío en los anillos de crecimiento (Mörath, 1931), las características de la estructura de la pared celular como el ángulo de las fibras (Frey y Wyssling, 1940), y las puntuaciones (Cockwell, 1946) y la composición química de la laminilla media (Frey y Wyssling, 1940).

\section{Materiales y Métodos}

La madera de E. tereticornis se obtuvo de una cortina de 50 años de edad ubicada en San Jorge, departamento de Durazno, perteneciente a un predio del Grupo Forestal San Gregorio. Se seleccionaron cinco árboles al azar, de los que se obtuvo una troza de $3,5 \mathrm{~m}$, de esta se sacaron dos discos de cada extremo, uno en altura 0 y otro a $3,5 \mathrm{~m}$ de altura (Figura 1). Los árboles tenían un promedio de diámetro máximo y mínimo en la altura basal de $542 \mathrm{~mm}$ y de $360 \mathrm{~mm}$, respectivamente. En altura los mismos diámetros eran de $440 \mathrm{~mm}$ y $319 \mathrm{~mm}$. La preparación de las probetas se realizó en el Departamento de Proyectos Forestales de LATU (DPF-LATU).

\section{Preparación de probetas}

Para la preparación, acondicionamiento y obtención de las probetas para el ensayo de contracción e hinchamiento se siguió la norma UNIT 363:2008.

De cada uno de los discos se obtuvieron prismas (o preprobetas) distribuidos en el radio, como se muestra en la Figura 1, desde una posición cercana a la médula hasta una cercana a la corteza.

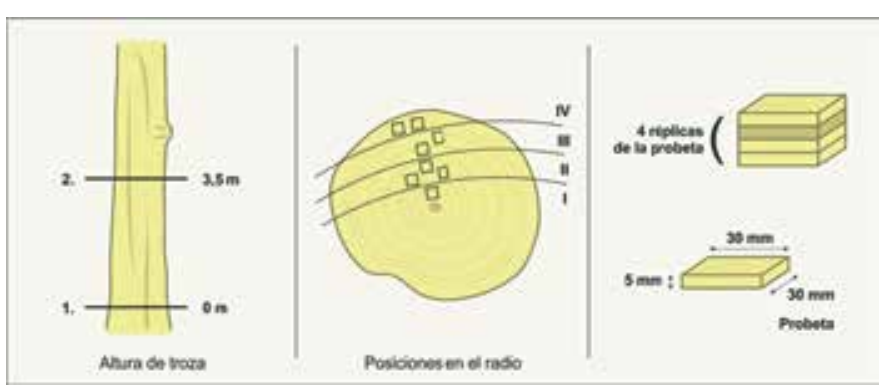

Figura 1. Detalle de la obtención de la troza y preparación de probetas.

Por las características que presentaban los árboles al formar parte de una cortina forestal sin manejo y ser especímenes adultos con madera de tensión y diferentes diámetros de sección, se dificultó la obtención de igual número de prismas en los radios. Los prismas fueron ubicados evitando defectos de la madera, tales como presencia de nudos, desviaciones en la fibra y madera de tensión.

A efectos de simplificar el análisis se dividió el radio en cuatro áreas, se contó el número de prismas incluidos en cada área y se trabajó con un resultado promedio de estos para cada propiedad. En la Tabla 2 se muestra el número de prismas por posición y altura tomadas.

\begin{tabular}{|c|c|c|}
\hline \multicolumn{3}{|c|}{ Número de prismas } \\
\hline \multirow{3}{*}{ ALTURA } & POSICIÓN & $\mathbf{N}^{\mathbf{0}}$ \\
\hline \multirow{3}{*}{1} & I & 9 \\
\cline { 2 - 3 } & II & 12 \\
\cline { 2 - 3 } & III & 8 \\
\cline { 2 - 3 } & IV & 10 \\
\hline \multirow{2}{*}{2} & SUB-TOTAL & 39 \\
\hline \multirow{2}{*}{} & I & 3 \\
\cline { 2 - 3 } & II & 11 \\
\cline { 2 - 3 } & III & 12 \\
\cline { 2 - 3 } & IV & 12 \\
\hline & SUB-TOTAL & 38 \\
\hline & TOTAL & 77 \\
\hline
\end{tabular}

Tabla 2. Número de prismas por posición en el radio de médula a corteza (I - IV) y por altura (1 y 2). 
Los prismas fueron sumergidos en agua hasta estar saturados completamente. Se sacaron cuatro probetas por prisma; estas réplicas son para evitar perder el dato en caso de que existiera colapso o se presentaran grietas. Las probetas tenían una dimensión de $30 \mathrm{~mm}$ de ancho, $30 \mathrm{~mm}$ de espesor y $5 \mathrm{~mm}$ de altura (Figura 1 ).

Como se aprecia en la Tabla 2, en la altura 1 se trabajó con un total de 39 prismas, en la altura 2 con 38 prismas, cada uno con 4 réplicas, lo que hace un total de 308 probetas medidas, distribuidas en las 4 posiciones y en las 2 alturas.

\section{Proceso de desorción de la madera}

El proceso de desorción de la madera consiste en la pérdida de humedad de la misma, bajo diferentes condiciones de humedad relativa y de temperatura del aire, lo que implica una variación en las dimensiones iniciales. Esta variación en las dimensiones se llama contracción (Kollmann y Coté, 1984).

A una misma temperatura si se baja la humedad relativa se logra ir disminuyendo el contenido de humedad de equilibrio de la madera (Kollmann y Coté, 1984).

Se utilizaron diferentes humedades relativas a una misma temperatura $\left(25^{\circ} \mathrm{C}\right)$, generando diferentes condiciones de humedades de equilibrio en la madera. Las humedades relativas variaron entre $84 \%, 76 \%, 63 \%$ y $53 \%$ como se puede ver en la Tabla 3 , teniendo en cuenta los diferentes ambientes en los que puede utilizarse la madera.

\begin{tabular}{|c|c|c|}
\hline $\begin{array}{l}\text { Humedad relativa a } \\
25^{\circ} \mathrm{C} \text { de temperatura }\end{array}$ & $\begin{array}{c}\text { Humedad de equilibrio } \\
\text { de la madera }\end{array}$ & Posibles usos \\
\hline $84 \%$ & $18 \%$ & \multirow{2}{*}{$\begin{array}{l}\text { Pisos, aberturas y muebles } \\
\text { en el exterior }\end{array}$} \\
\hline $76 \%$ & $15 \%$ & \\
\hline $63 \%$ & $12 \%$ & \multirow{2}{*}{$\begin{array}{l}\text { Pisos, aberturas y muebles } \\
\text { en el interior }\end{array}$} \\
\hline $53 \%$ & $8 \%$ & \\
\hline
\end{tabular}

Tabla 3. Condiciones de humedad relativa, humedad de equilibrio y posibles usos.

Se realizaron mediciones de las dimensiones en sentido radial y tangencial con micrómetro digital Sony DZ-501 y se tomó el peso en balanza analítica de cada una de las probetas en estado verde y en cada uno de los estados de equilibrio a la temperatura de $25^{\circ} \mathrm{C}$.

Para evitar el colapso de las probetas, luego de las mediciones en estado verde éstas se fueron secando gradualmente. Este secado consistió en la disminución de la humedad inicial (verde) de las probetas hasta un contenido de humedad aproximado de $26 \%$, para evitar la pérdida vertiginosa del agua que se ocasionaría si se colocaran directamente a condiciones de $18 \%$ de humedad de equilibrio. Esto se realizó en un ambiente cerrado con presencia de bandejas de agua que aseguraban una humedad relativa alta en el ambiente.

Cuando el contenido de humedad aproximado fue de $26 \%$, se colocaron las probetas en la cámara de acondicionamiento Advantec AG-327 en condiciones iniciales de $25{ }^{\circ} \mathrm{C}$ de temperatura y $84 \%$ de humedad relativa. Para controlar las condiciones de equilibrio se utilizaron probetas testigo que fueron pesadas hasta que llegaron a un peso constante, infiriendo así que todas las probetas alcanzaron el contenido de humedad de equilibrio en las condiciones establecidas.

\section{Proceso de sorción de la madera}

El proceso de sorción consiste en la absorción de humedad por la madera hasta un estado de equilibrio con las condiciones de temperatura y humedad relativa del aire, lo que implica un cambio en las dimensiones de las probetas que se denomina hinchamiento (Kollmann y Coté, 1984).

Una vez terminado el proceso de desorción se comenzó el proceso de aumento de la humedad relativa ambiente en la misma cámara. Se tomaron registros de las dimensiones y el peso de las probetas en las condiciones de equilibrio $63 \%, 76 \%$ y $84 \%$ de humedad relativa a la misma temperatura de $25^{\circ} \mathrm{C}$. Las determinaciones se realizaron de la misma forma que en el proceso de desorción.

La Tabla 4 presenta los tiempos que demoraron las probetas en alcanzar las condiciones de equilibrio determinadas para ambos procesos.

\begin{tabular}{|c|c|c|}
\cline { 2 - 3 } \multicolumn{1}{c|}{} & $\begin{array}{c}\text { Humedad relativa a } \\
\mathbf{2 5}^{\mathbf{0}} \mathbf{C} \text { de temperatura }\end{array}$ & $\begin{array}{c}\text { Tiempo (días) en alcanzar } \\
\text { la condición de equilibrio }\end{array}$ \\
\hline $\begin{array}{c}\text { Proceso } \\
\text { de } \\
\text { desorción }\end{array}$ & $84 \%$ & 7 \\
\hline $76 \%$ & 28 \\
\hline $\begin{array}{c}\text { Proceso } \\
\text { de } \\
\text { sorción }\end{array}$ & $63 \%$ & 40 \\
\hline & $53 \%$ & 62 \\
\hline
\end{tabular}

Tabla 4. Condiciones de humedad relativa, temperatura y tiempos de duración para los procesos de sorción y desorción.

\section{Peso específico aparente (PEA) y contenido de humedad}

El peso específico aparente básico se determina como la relación entre el peso anhidro de cada probeta y su volumen en estado verde según la norma ASTM D2395, Método B. Se calculó también el peso específico aparente en las condiciones de $15 \%$ y $12 \%$ de humedad de equilibrio según la norma ASTM D2395, Método A.

El contenido de humedad se determinó para cada una de las probetas en cada uno de los estados de equilibrio según ASTM D4442, Método B.

\section{Contracción e hinchamiento lineal y volumétrico}

La contracción lineal se define como el cociente entre la diferencia de dimensiones lineales de un estado a otro de menor humedad y la dimensión en estado saturado. El hinchamiento lineal relaciona la diferencia de dimensiones lineales de un estado a otro de mayor humedad con la misma dimensión en estado anhidro (Coronel, 1994).

Para la determinación de los valores de contracción e hinchamiento de la madera se utilizan las dimensiones en sentido radial y tangencial de las probetas. Se calculan valores para cada uno de los estados de equilibrio en los procesos de desorción y sorción.

Para los cálculos de la contracción $(\mathrm{C})$ y el hinchamiento $(\mathrm{H})$ volumétrico se utiliza la fórmula citada en Coronel (1994) y Kollmann y Coté (1984):

$$
C=1-(1-C t)^{*}(1-C r)^{*}(1-C l)
$$

Donde $\mathrm{Ct}$, $\mathrm{Cr}$ y $\mathrm{Cl}$ son contracción tangencial, radial y longitudinal, respectivamente.

$$
H=(1+H t)^{*}(1+H r)^{*}(1+H l)-1
$$

Donde $\mathrm{Ht}, \mathrm{Hr}$ y $\mathrm{Hl}$ son hinchamiento tangencial, radial y longitudinal, respectivamente.

La variación dimensional en el sentido longitudinal para la contracción y el hinchamiento se considera despreciable (Kollmann y Coté, 1984; Coronel, 1994). 


\section{Isoterma higroscópica a $25^{\circ} \mathrm{C}$}

Para construir la isoterma higroscópica se fueron variando las condiciones de humedad relativa del aire manteniendo constante la temperatura a $25^{\circ} \mathrm{C}$.

\section{Análisis de datos}

Para el análisis de los resultados se trabajó con las observaciones que se encontraron dentro de tres desvíos respecto a la media (ISO 7870-1:1993). Los datos que quedaron fuera de los límites coincidieron con probetas que presentaron colapso.

La estadística descriptiva de los datos para caracterizar la madera fue realizada en planilla de cálculo Excel. Para el análisis en el radio y en altura se realizó una prueba de comparación de medias de Tukey, con un nivel de significación al 5\% utilizando el programa estadístico InfoStat 2011.

Se determinó el PEA básico al 12\% y al 15\%, de manera de caracterizar la madera en estudio y comparar los resultados obtenidos con otros trabajos. Se analizó el PEA básico entre las dos alturas, y en el radio para las dos alturas.

Se determinó la contracción e hinchamiento máximo radial y tangencial de la madera en la altura y en el radio. Además, para las diferentes humedades de equilibro obtenidas se determinó la contracción y el hinchamiento radial y tangencial.

Se correlacionó gráficamente el contenido de humedad de equilibrio y la humedad relativa del ambiente presentando parte de la isoterma higroscópica a $25^{\circ} \mathrm{C}$.

Por último se calcularon los coeficientes de anisotropía para la contracción y el hinchamiento, así como el coeficiente de histéresis de la madera para compararla con otras.

\section{Resultados y Discusión}

\section{Peso específico aparente}

La madera presentó un peso específico aparente básico promedio de 0,630 con un desvío estándar de 0,07. Si se compara con resultados publicados en Australia por Kingston y Risdon (1961) y por Bootle (1983), quienes establecieron valores de 0,780 y 0,900 , respectivamente, los obtenidos en el trabajo son menores. El resultado es similar al obtenido por Mantero (1999) de 0,680 con un desvío estándar de 0,07.

Se obtuvieron los resultados del peso específico para valores de contenido de humedad de $12 \%$ y $15 \%$ de 0,859 y 0,872 . Si se los compara con valores presentados por Boas $(1947)$ al $12 \%(0,977)$ y por el Instituto de Pesquisas Tecnológicas de Brasil (1956) al 15\% $(0,950)$ estos son menores. Si se compara con datos publicados en Uruguay por Tuset y García Taibo (1987) al 12\% $(0,820)$ y por Mantero (1999), de 0,830 , los resultados obtenidos en este trabajo fueron mayores.

Se analizó el PEA básico para las dos alturas y no se encontraron diferencias significativas al 5\%.

En el análisis del PEA en el radio a las dos alturas se observó diferencias significativas entre las posiciones, como se muestra en los Gráficos 1 y 2. Para la altura basal (1) la posición mas cercana a la médula fue la única que presentó diferencia con respecto a las demás posiciones (II, III y IV). Para la altura 2 se observó que entre la posición I y II no hubo diferencias significativas, pero si existió diferencia entre la posición I en comparación con las III y IV.

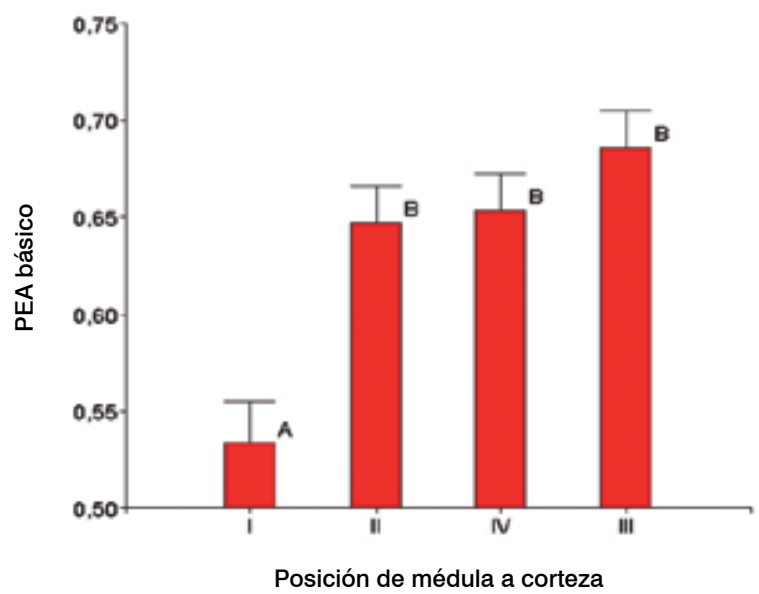

Gráfico 1. Comparación de las medias del peso específico aparente básico en las cuatro posiciones en el radio para la altura 1.

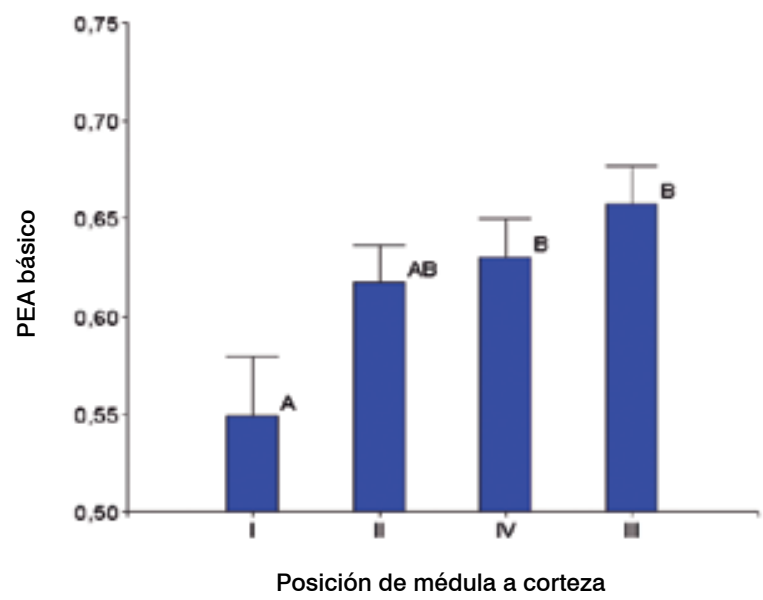

Gráfico 2. Comparación de las medias del peso específico aparente básico en las cuatro posiciones en el radio para la altura 2.

En las dos alturas, el peso específico de la madera es mayor en la posición cercana a la corteza.

\section{Contenido de humedad de equilibrio (CHE)}

Con respecto a las condiciones de contenido de humedad de equilibrio alcanzadas en las diferentes humedades relativas (HR), estos resultados se presentan en la Tabla 5. 


\begin{tabular}{|c|c|c|c|c|c|c|c|}
\hline \multirow[b]{2}{*}{$\mathrm{HR}$ a $25^{\circ} \mathrm{C}$} & \multicolumn{4}{|c|}{ Proceso de desorción } & \multicolumn{3}{|c|}{ Proceso de sorción } \\
\hline & $84 \%$ & $76 \%$ & $63 \%$ & $53 \%$ & $63 \%$ & $76 \%$ & $84 \%$ \\
\hline & \multicolumn{7}{|c|}{ CHE (\%) } \\
\hline Máximo & 26,6 & 21,6 & 16,5 & 14,3 & 15,4 & 17,7 & 19,8 \\
\hline Mínimo & 21,5 & 17,9 & 13,9 & 12,1 & 13,1 & 15,1 & 16,9 \\
\hline Promedio & 23,6 & 19,8 & 15,1 & 13,0 & 14,1 & 16,4 & 18,3 \\
\hline Desvío estándar & 1,14 & 0,69 & 0,49 & 0,48 & 0,47 & 0,50 & 0,53 \\
\hline Coef. de variación (\%) & 4,83 & 3,50 & 3,26 & 3,66 & 3,36 & 3,03 & 2,90 \\
\hline
\end{tabular}

Tabla 5. Contenido de humedad de equilibrio (\%) para las diferentes condiciones de humedad relativa (\%) en ambos procesos.

\section{Contracción e hinchamiento para E. tereticornis}

Se analizó la contracción y el hinchamiento máximo para la madera y los resultados se expresan en la Tabla 6.

\begin{tabular}{|c|c|c|c|c|c|c|}
\hline \multirow{2}{*}{} & \multicolumn{3}{|c|}{ Contracción máxima (\%) } & \multicolumn{3}{c|}{ Hinchamiento máximo (\%) } \\
\cline { 2 - 8 } & Radial & Tangencial & Volumétrica & Radial & Tangencial & Volumétrica \\
\hline Máximo & 10,68 & 28,22 & 33,12 & 11,95 & 39,31 & 49,52 \\
\hline Mínimo & 4,03 & 5,06 & 12,24 & 4,20 & 5,32 & 13,95 \\
\hline Promedio & 7,04 & 14,70 & 20,64 & 7,61 & 17,61 & 26,62 \\
\hline Desvío estándar & 1,60 & 4,72 & 5,44 & 1,86 & 6,80 & 9,04 \\
\hline Coef. de variación (\%) & 22,69 & 32,08 & 26,36 & 24,39 & 38,60 & 33,94 \\
\hline
\end{tabular}

Tabla 6. Resumen de contracción e hinchamiento máximo, lineal y volumétrico.

Se comparan estos resultados con los presentados por Coronel (1994) para la misma especie. Los valores que obtiene este último son 7,77\% y $10,53 \%$ para contracción radial y tangencial, respectivamente, con coeficientes de variación similares respecto a los resultados obtenidos. Los valores para el hinchamiento radial y tangencial según Coronel (1994) son 6,74 \% y 11,88 \%, respectivamente. Tanto para la contracción como el hinchamiento los resultados obtenidos por Coronel (1994) para madera argentina son menores. Se compararon los resultados de la Tabla 6 con los obtenidos por Mantero (1999), quien obtuvo valores para madera nacional de 7,77\% para contracción radial y 13,62\% para contracción tangencial, presentando valores de desvíos similares.

La variación en altura de la contracción e hinchamiento lineal solo mostró diferencias significativas en el sentido radial. La altura 1 (basal) presentó una menor contracción e hinchamiento radial que la altura 2. Este comportamiento difiere de lo indicado por Coronel (1994) y de los resultados que presentó Calvo (2006) para E. grandis de 17 años, en donde la tendencia del hinchamiento tanto radial como tangencial es de disminuir con la altura.

Se analizó la contracción y el hinchamiento en el sentido radial y tangencial desde una posición cercana a la médula hasta una cercana a la corteza, para las dos alturas. Los resultados se incluyen en la Tabla 7.

Para ambas alturas, la contracción y el hinchamiento lineal disminuyen hacia la posición mas cercana a la corteza. Esto difiere de lo indicado por Coronel (1994) pero es similar al comportamiento que presentaron los árboles de 42 años de E. bosistoana según Cardoso y Castagna (2012).

Se analizaron los valores de contracción e hinchamiento lineal para cada uno de los contenidos de humedad de equilibrio alcanzados. Los resultados se presentan en las Tablas 8 y 9.

Se calcularon los coeficientes de anisotropía como la relación entre la variación tangencial y la radial máxima tanto para la contracción, que fue 2,1, como para el hinchamiento, que fue 2,3. Se calculó el coeficiente de anisotropía para las diferentes humedades de equilibrio alcanzadas. Los resultados se encuentran en la Tabla 10. El coeficiente de anisotropía mide la variación dimensional tangencial respecto a la radial siendo uno el valor ideal. 


\begin{tabular}{|c|c|c|c|c|}
\hline ALTURA 1 & HT & CT & HR & CR \\
\hline I & $29,89 \mathrm{a}$ & $22,77 \mathrm{a}$ & $8,97 \mathrm{a}$ & $8,22 \mathrm{a}$ \\
\hline II & $19,75 \mathrm{~b}$ & $16,34 \mathrm{~b}$ & $7,50 \mathrm{ab}$ & $6,97 \mathrm{ab}$ \\
\hline III & $15,17 \mathrm{bc}$ & $13,15 \mathrm{bc}$ & $6,29 \mathrm{bc}$ & $5,91 \mathrm{bc}$ \\
\hline IV & $11,48 \mathrm{c}$ & $10,27 \mathrm{c}$ & $5,19 \mathrm{c}$ & $4,93 \mathrm{c}$ \\
\hline ALTURA 2 & HT & CT & HR & CR \\
\hline I & $26,25 \mathrm{a}$ & $20,78 \mathrm{a}$ & $9,98 \mathrm{a}$ & $9,07 \mathrm{a}$ \\
\hline II & $21,35 \mathrm{a}$ & $17,47 \mathrm{a}$ & $9,63 \mathrm{ab}$ & $8,77 \mathrm{ab}$ \\
\hline III & $15,21 \mathrm{~b}$ & $13,14 \mathrm{~b}$ & $8,09 \mathrm{bc}$ & $7,43 \mathrm{bc}$ \\
\hline IV & $11,66 \mathrm{~b}$ & $10,39 \mathrm{~b}$ & $6,64 \mathrm{c}$ & $6,22 \mathrm{c}$ \\
\hline
\end{tabular}

Tabla 7. Contracción e hinchamiento lineal promedio (\%) por posición en el radio para las dos alturas. Las medias con una letra común no son significativamente diferentes $(p<=0,05)$.

\begin{tabular}{|c|c|c|c|c|c|c|c|c|}
\hline \multirow{2}{*}{ CONTRACCIÓN } & \multicolumn{2}{|c|}{ CHE 13\% } & \multicolumn{2}{|c|}{ CHE 15\% } & \multicolumn{2}{|c|}{ CHE 20\% } & \multicolumn{2}{|c|}{ CHE 24\% } \\
\hline & Rad & $\operatorname{Tg}$ & Rad & $\operatorname{Tg}$ & Rad & $\mathrm{Tg}$ & Rad & $\operatorname{Tg}$ \\
\hline Promedio & 4,53 & 11,30 & 4,07 & 10,59 & 3,41 & 9,50 & 2,79 & 8,58 \\
\hline Desvío estándar & 1,50 & 4,86 & 1,51 & 4,92 & 1,59 & 5,31 & 1,49 & 5,19 \\
\hline
\end{tabular}

Tabla 8. Valores promedio de contracción radial y tangencial (\%) para los diferentes contenidos de humedad de equilibrio.

\begin{tabular}{|c|c|c|c|c|c|c|c|c|}
\hline \multirow{2}{*}{ HINCHAMIENTO } & \multicolumn{2}{|c|}{ CHE $13 \%$} & \multicolumn{2}{|c|}{ CHE $14 \%$} & \multicolumn{2}{|c|}{ CHE 16\% } & \multicolumn{2}{|c|}{ CHE $18 \%$} \\
\hline & Rad & $\operatorname{Tg}$ & Rad & $\mathrm{Tg}$ & Rad & $\mathrm{Tg}$ & Rad & $\mathrm{Tg}$ \\
\hline Promedio & 2,70 & 3,99 & 2,97 & 4,39 & 3,54 & 5,46 & 4,03 & 6,48 \\
\hline Desvío estándar & 0,38 & 0,40 & 0,41 & 0,39 & 0,48 & 0,46 & 0,55 & 0,62 \\
\hline
\end{tabular}

Tabla 9. Valores promedio de hinchamiento radial y tangencial (\%) para los diferentes contenidos de humedad de equilibrio.

\begin{tabular}{|c|c|c|c|}
\hline CHE (\%) & $\begin{array}{c}\text { Coef. anisotropía para } \\
\text { la contracción }\end{array}$ & CHE (\%) & $\begin{array}{c}\text { Coef. anisotropía para } \\
\text { el hinchamiento }\end{array}$ \\
\hline 24 & 3,06 & 18 & 1,60 \\
\hline 20 & 2,77 & 16 & 1,54 \\
\hline 15 & 2,60 & 14 & 1,48 \\
\hline 13 & 2,50 & 13 & 1,47 \\
\hline
\end{tabular}

Tabla 10. Coeficiente de anisotropía para la contracción y el hinchamiento para cada contenido de humedad de equilibrio alcanzado. 


\section{Isoterma higroscópica para $E$. tereticornis}

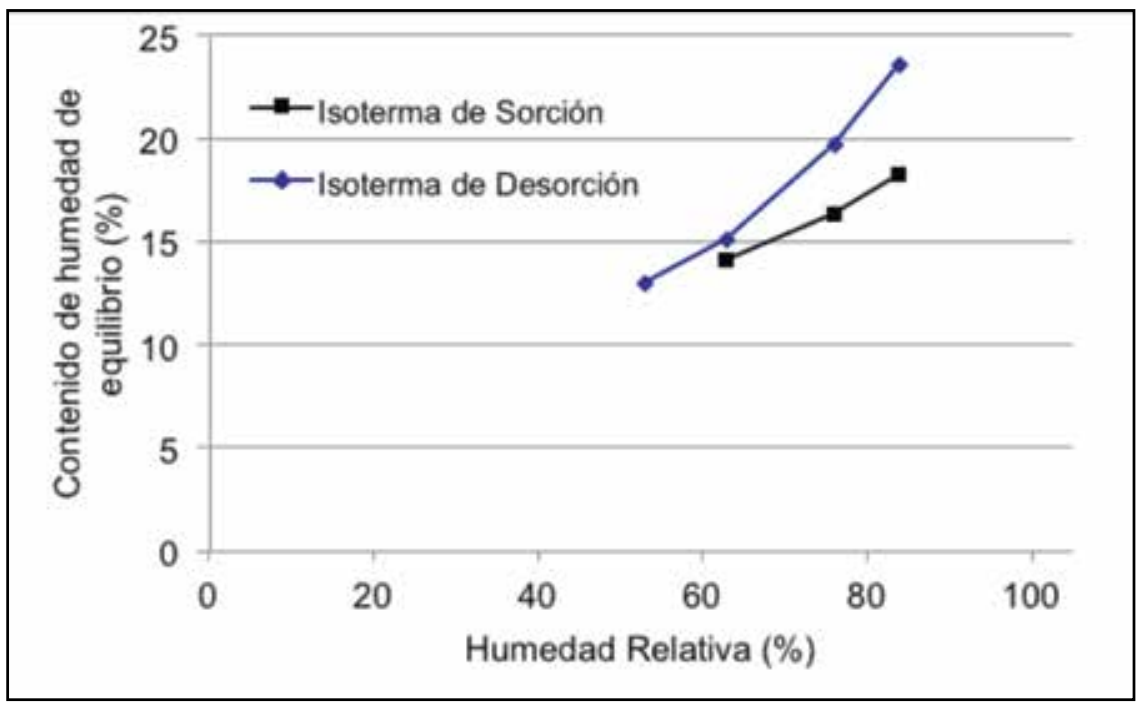

Gráfico 3. Isoterma higroscópica parcial en $25^{\circ} \mathrm{C}$ para la madera de E. tereticornis.

Se observó una diferencia entre la curva de sorción y la de desorción para la misma temperatura. La histéresis de la madera se considera como la diferencia en contenido de humedad entre la isoterma de desorción y sorción (Kollmann y Coté, 1984). La histéresis es constante a una humedad relativa dada (García, 1990). Coronel (1994) define el coeficiente de histéresis como la relación entre el contenido de humedad de equilibrio de sorción y desorción a una humedad relativa de $85 \%$. Se observó un coeficiente de histéresis de 0,8 para la madera en estudio. Se encontraron valores de referencia de este coeficiente para otras maderas duras en un trabajo realizado por Frank et al. (2002) donde menciona para Prosopis alba (Algarrobo blanco) y Aspidosperma quebracho-blanco (Quebracho blanco) coeficientes de 0,41 y 0,91 , respectivamente. García et al (2004) encontraron un coeficiente de 0,87 para el Pinus sylvestris $L$.

En el proceso de pérdida y ganancia de humedad en la madera se observó una diferencia de las humedades de equilibrio que se hace mayor en la medida que aumenta la humedad relativa. Para una humedad relativa de $63 \%$, la madera presentó una diferencia en el contenido de humedad de equilibrio de $1 \%$ entre ambos procesos. La diferencia fue de $3,4 \%$ y $5,3 \%$ para $76 \%$ y $84 \%$ de humedad relativa.

\section{Conclusiones}

La variación de la contracción y el hinchamiento lineal en el sentido radial del árbol disminuye en la madera más cercana a la corteza, que es la que presentó mayor peso específico aparente. En el análisis en altura no presentaron diferencias significativas las propiedades de contracción e hinchamiento tangencial y peso específico aparente básico, aunque si la contracción e hinchamiento en sentido radial. Se podría esperar que este comportamiento se explicara por las características anatómicas que podría presentar la madera de árboles de más de 50 años de E. tereticornis, como el ángulo de la fibrilla u otras características de la pared secundaria. Esto se debería de corroborar con otros estudios para esta especie.

Según la clasificación realizada por Coronel (1994) para maderas argentinas, la madera estudiada se clasifica como "no apta para varios usos", dado que presenta un coeficiente de anisotropía de 2,1. Según Etchenique et al. (1975), esta madera está incluida en la categoría de alto índice para distorsiones y alabeos. Ordóñez et al. (1990) clasifican a la madera como moderadamente estable dimensionalmente.

A pesar de tener 2,1 de coeficiente anisotrópico para la contracción y 2,3 para el hinchamiento, la clasificación no especifica los usos a los cuales hace mención. Es posible utilizar esta madera para algunos productos como pisos de alto tránsito, mobiliario de exterior y cubiertas, productos en los cuales esta anisotropía puede ser tratada por diseño.

Como era esperable, la madera de E. tereticornis presenta porcentajes de contracción e hinchamiento comparables con las maderas "nerviosas" o de grandes movimientos. Esto motiva a continuar con los estudios a contenidos de humedad relativa más bajos, completando la isoterma de $25^{\circ} \mathrm{C}$ y generando la información necesaria para el conocimiento de esta madera, mejorando sus usos, por ejemplo, en muebles y aberturas.

Este estudio se enfocó en el análisis de un ciclo en el que la madera cedió agua al medio ambiente, contrayéndose, y luego absorbió agua, hinchándose. Es de interés en un próximo estudio continuar con los ciclos de fatiga de la madera, analizando su respuesta.

\section{Reconocimientos}

Al Ing. Agr. Alfredo Clement (Grupo Forestal San Gregorio) por proveernos la madera para este estudio.

A los compañeros del Departamento de Proyectos Forestales de LATU.

\section{Referencias}

\section{- AMERICAN SOCIETY FOR TESTING AND MATERIALS}

(Estados Unidos). ASTM D4442: Test methods for direct moisture content measurement of wood and wood-base materials. Baltimore: ASTM, 2002

- AMERICAN SOCIETY FOR TESTING AND MATERIALS

(Estados Unidos). ASTM D2395: Test methods for specific gravity of wood and wood-base materials. Baltimore: ASTM, 2002

- BOAS, I.R. The commercial timbers of Australia; their properties and uses. Melbourne: CSIRO, 1947.

- BOOTLE, K. R. Wood in Australia; types, properties and uses. Sydney: McGraw-Hill, 1983. ISBN 0074510479.

- CALVO, A.F.; CORTINA, A.D.; CUFFRÉ, A.G.; PITER, J.C.; STEFANI, P.M.; TORRÁN, E.A. Variación radial y axial del 
hinchamiento, del factor anisotrópico y de la densidad, en el Eucalyptus grandis de Argentina. En: Maderas. Ciencia y Tecnología. 2006, 8(3):159-168.

- CARDOSO, A.; CASTAGNA, A. Caracterización de las propiedades físicas y mecánicas de la madera de una población de Eucalyptus bosistoana cultivado en Uruguay. Montevideo: Facultad de Agronomía, 2012.

- COCWELL, A. Influence of fibril angle on longitudinal shrinkage of ponderosa pine wood. En: J. Forestry. 1946, 44:876-878. Citado en: KOLLMANN, F.; COTÉ, W. Principles of wood science and technology. V. 1. 2da ed. Berlin: Springer Verlag, 1984. 592 p. ISBN 0-387-04297-0

- CORONEL, E. Fundamentos de las propiedades físicas y mecánicas de las maderas; aspectos teóricos y prácticos para la determinación de las propiedades y sus aplicaciones. Santiago del Estero: El Liberal, 1994. ISBN: 950-43-6061-0

- ETCHENIQUE-MANRIQUE, R. [et al.] Caracterización tecnológica de diez especies. Estudio botánico y ecológico de la región de Uxanapa México: INIREB-P.N.I.E.T. ; CONACYT, 1975. pp.6165. Citado en: FUENTES-TALAVERA, F. J.; SILVIA-GUZMÁN, J. A.; LOMELÍ-RAMÍREZ, M.G.; RICHTER, H.G.; SANJUÁNDUEÑAS, R. Comportamiento higroscópico de la madera de Persea americana var. guatemalensis Mill (Hass). En: Revista Chapingo Serie Ciencias Forestales y del Ambiente. 2002, 8(1):49-56.

- FRANK, W.; SANABRIA, E.O.; PAZ, J. M. El polietilenglicol y las variaciones en las propiedades mecánicas de Aspidosperma quebracho-blanco Schlecht [En línea]. Corrientes: Facultad de Agroindustrias-UNNE, 2002. [Consulta: 4 de octubre de 2013]. Disponible en: http://www.unne.edu.ar/unnevieja/Web/cyt/ cyt/2002/07-Tecnologicas/T-044.pdf

- FREY-WYSSLING, A. Die Anisotropie des Schwindmabes auf dem Holzquerschnitt. En: Holz als Roh- und Werkstoff. 1940, 3:43-45. Citado en: KOLLMANN, F.; COTÉ, W. Principles of wood science and technology. V. 1. 2da ed. Berlin: Springer Verlag, 1984. 592 p. ISBN: 0-387-04297-0

- FUENTES-TALAVERA, F. J.; SILVIA-GUZMÁN, J. A.; LOMELÍRAMÍREZ, M.G.; RICHTER, H.G.; SANJUÁN-DUEÑAS, R. Comportamiento higroscópico de la madera de Persea americana var. guatemalensis Mill (Hass). En: Revista Chapingo Serie Ciencias Forestales y del Ambiente. 2002, 8(1):49-56.

- FWPA, WOOD SOLUTIONS. Forest-red-gum/Eucalyptus tereticornis. [En línea]. Melbourne: FWPA, 2013. [Consulta: 8 marzo 2013]. Disponible en: http://www.woodsolutions.com.au/WoodSpecies/forest-red-gum.

- GARCÍA, ESTEBAN L. Fatiga higroscópica y dimensional de la madera. Madrid: Departamento de Ingeniería Forestal de la Escuela Técnica superior de Ingeniería de Montes. Universidad Politécnica de Madrid, 1990. (Tesis de Doctorado)

- GARCÍA, ESTEBAN L.; GUINDEO CASASÚS, A.; DE PALACIOS, P.; GARCÍA FERNÁNDEZ, F. Histéresis de la madera de Pinus sylvestris L. para isotermas de $35^{\circ} \mathrm{C}$ mediante el método de sales saturadas. En: Revista Materiales de Construcción. 2004, 54(276):51-64

- INSTITUTO DE PESQUISAS TECNOLÓGICAS. Tabelas de resultados obtidos para madeiras nacionais. En: Boletim Técnico. 1956, (31):62

- INSTITUTO URUGUAYO DE NORMAS TÉCNICAS (Uruguay). UNIT 363: Maderas; determinación de la contracción. Montevideo: UNIT, 2008.

- INTERNATIONAL STANDARD ORGANIZATION (Suecia). ISO 7870-1: Control charts - General guide and introduction. Suecia: ISO, 1993.

- KINGSTON, R.; RISDON, J. Shrinkage and density of australian and other South-West Pacific woods. Clayton South: CSIRO. Forest Products Division, 1961. (Technical Paper; 13)

- KOLlMANN, F.; COTÉ, W. Principles of wood science and technology. V. 1. 2da ed. Berlin: Springer Verlag, 1984. 592 p. ISBN: 0-387-04297-0

- MANTERO, Carlos. Propiedades físicas y mecánicas de los eucaliptos: E. grandis; E. globulus; E. tereticornis. En: Uruguay Forestal. 1999, 21(9):13-16.

- MGAP-DGF. Actualización de la cartografía forestal del Uruguay [En línea]. Montevideo: DGF, 2012. [Consulta: 8 de marzo de 2013].
Disponible en: http://www.mgap.gub.uy/portal/hgxpp001.aspx?7,20, 441,O,S,0,MNU;E;134;2;MNU

- MÖRATH, E. Kolloidchem. En: Beihefte. 1931, 33:131. Citado en: KOLLMANN, F.; COTÉ, W. Principles of wood science and technology. V. 1. 2da ed. Berlin: Springer Verlag, 1984. 592 p. ISBN: 0-387-04297-0

- ORDÓÑEZ, C.V. R.; BÁCENAS, P., G. M.; QUIRÓZ, A.

Características físico-mecánicas de la madera de diez especies de San Pablo Macuiltianguis, Oax. La madera y su Uso. San Pablo: Instituto de Ecología, 1990. Citado en: FUENTES-TALAVERA, F. J.; SILVIA-GUZMÁN, J. A.; LOMELÍ-RAMÍREZ, M.G.; RICHTER, H.G.; SANJUÁN-DUEÑAS, R. Comportamiento higroscópico de la madera de Persea americana var. guatemalensis Mill (Hass). En: Revista Chapingo Serie Ciencias Forestales y del Ambiente. 2002, 8(1):49-56.

- TUSET, R.; GARCIA TAIBO, R. Producción de durmientes de eucalipto. Montevideo: Facultad de Agronomía, UdelaR, 1987. (Boletín de Investigación; 4)

- UNIVERSIDAD NACIONAL DE CÓRDOBA. Infostat. Versión 2011. Córdoba: UNC, 2011. 